Revue internationale de l'économie sociale

Recma

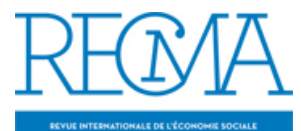

\title{
Temps forts
}

\section{Jordane Legleye, Patricia Toucas-Truyen et João Salazar Leite}

Numéro 328, avril 2013

URI : https://id.erudit.org/iderudit/1015522ar

DOI : https://doi.org/10.7202/1015522ar

Aller au sommaire du numéro

Éditeur(s)

Association Recma

ISSN

1626-1682 (imprimé)

2261-2599 (numérique)

Découvrir la revue

Citer ce document

Legleye, J., Toucas-Truyen, P. \& Leite, J. S. (2013). Temps forts. Revue internationale de l'économie sociale, (328), 7-15.

https://doi.org/10.7202/1015522ar d'utilisation que vous pouvez consulter en ligne.

https://apropos.erudit.org/fr/usagers/politique-dutilisation/ 


\section{TEMPS FORTS}

\section{Sortir de la crise de la pensée économique}

L’Association française d'économie politique (Afep) est à l'initiative d'un appel lancé avec des philosophes, des sociologues, des historiens, des juristes, des spécialistes des sciences de gestion et des sciences politiques pour réclamer la création d'une nouvelle section d'économie au Conseil national des universités (CNU). Les accents de cet appel ne sont pas sans rappeler les textes fondateurs de la Recma ${ }^{(1)}$.

\section{Pour une $78^{\mathrm{e}}$ section}

\section{«Economie et société " au CNU}

"Les signataires de ce texte considèrent que la situation actuelle ne révèle pas seulement une crise de l'économie, mais également une crise intellectuelle profonde, celle de la pensée économique. Les causes de cette crise sont nombreuses, et les solutions à y apporter se situent entre autres dans l'inventivité théorique et pratique. Mais nous souhaitons pointer ici une cause institutionnelle qui bloque cette inventivité et à laquelle une réponse politique, simple et rapide, pourrait être apportée. Une solution qui redonnerait immédiatement du souffle à la réflexion sur les affaires économiques et sociales de notre temps. [...] La pensée économique s'est progressivement nécrosée au fur et à mesure qu'elle s'écartait de sa posture originale de "science sociale" pour se calquer artificiellement sur les pratiques, les modalités d'organisation et de formalisation et sur les critères de scientificitésupposés être ceux des "sciences normales" fondées surl'existence de régularités naturelles. [...] La réalitédu paysage scientifique actuel des économistes, en France, mais aussi au niveau international, est celle d'un rétrécissement considérable des méthodes et des concepts tolérés par

(1) Consultable sur www.recma.org. la communauté scientifique comme "véritablement scientifiques". Les cadres du milieu universitaire (les "professeurs des universités"), qui encadrent des thèses, président des jurys, dirigent des masters ou des laboratoires, appartiennent presque tous aujourd'hui à un mainstream fondé sur un mixte constituéde méthodes mathématiques (assorties fréquemment d'une validation économétrique) et de la conceptualisation abstraite d'un homo œconomicus maximisateur ayant vocation, a priori, à expliquer toutes les interactions sociales. Faute d'une réforme institutionnelle, à l'horizon d'une dizaine d'années, la profession des économistes en France ne produira plus aucun économiste s'inspirant de la solide et longue tradition de l'économie politique (classique, marxiste, keynésienne, institutionnaliste, hayékienne, walrassienne, etc.).

Ce constat, longuement étayé depuis deux ans [...], nous conduit à demander la création, au sein de l'institution qui organise la formation supérieure et la recherche en France, le Conseil national des universités (CNU), d'une nouvelle section intitulée "Economie et société". La création d'une soixantedix-huitième section du CNU, qui relève d'une décision ministérielle, ne ferait sans doute pas grand bruit et ne constituerait certes pas en soi une révolution. Mais elle redonnerait immédiatement de l'oxygène à une pensée sur l'économie qui en a cruellement besoin. Elle fournirait les conditions institutionnelles pour qu'un vrai pluralisme existe dans la vie des idées, dans la pensée économique et sociale et, in fine, dans les pratiques de l'économie dans la société. »

\section{Un « programme général} de reconstitution sociale "

En octobre 1921, dans le premier numéro de la Revue des études coopératives, devenue la Recma, plusieurs centaines d'intellectuels, comme Charles Andler, Elie Halévie, 
Ernest Lavisse, Léon Brunschvicg, Lucien Lévi-Bruhl, Marcel Mauss, François Simiand, Charles Seignobos, Bernard Lavergne, Ernest Poisson..., signaient le Manifeste coopératif dont est tiré le présent intertitre. Celui-ci précédait un texte de Charles Gide, aux échos éminemment contemporains (2) (à condition de remplacer " coopération " par " économie sociale»), intitulé « Pourquoi les économistes n'aiment pas la coopération », qui se conclut par ces mots: "Les économistes veulent bien des sociétés coopératives, mais ils ne veulent pas du coopératisme, au sens de programme de transformation sociale, tel que cette revue a précisément pour but de l'enseigner et de le propager. [...] Si économistes et coopératistes ne s'aiment guère, c'est moins par opposition de doctrines que par incompatibilité d'esprit. Nous ne sommes pas de la même religion, ou plutôt nous ne sommes pas du même monde, au sens "mondain" de ce mot. Nous ne fréquentons pas la même société. L'école libérale est plus conservatrice encore que libérale, en ce sens que la libre concurrence qu'elle réclame ne lui apparaît désirable qu'autant qu'elle a pour résultat de maintenir l'ordre économique existant dans ses grandes lignes. Elle croit sincèrement que l'existence de la société est liée à celle de la société bourgeoise. Et toute action qui tend à la transformer, alors même qu'elle n'a recours pour cela qu'à l'association libre, telles le coopératisme ou le syndicalisme, lui apparaît comme néfaste. Les coopérateurs ne sont pas des révolutionnaires. Ils ne cherchent pas de solution en dehors des loiséconomiques, mais ils ne craignent pas desuivrecelles-cijusqu'au bout, si loin qu'elles puissent les mener.»

JORDANE LEGLEYE

\section{Un colloque sur l'ESS à Bercy}

Le 5 février 2013, le ministère de l'ESS et de la Consommation organisait un colloque à

(2) Voir l'étude de P. Frémeaux dans ce même numéro. l'intitulé ambitieux, "Penser et construire de nouveaux référentiels pour construire les politiques économiques de demain », qui réunissait un panel d'économistes « hétérodoxes » dans les locaux austères du ministère des Finances.

\section{Un sombre constat}

Dans son propos introductif, l'anthropologue Paul Jorion, spécialiste de la finance, a rappelé que le système darwinien appliqué à l'économie depuis le XIX siècle, avec pour conséquence la concentration des richesses, a impacté de façon désastreuse le fonctionnement de la démocratie. Or, "la démocratie est adaptative, mais le capitalisme qui caractérise son économie n'est pas, lui, adaptatif ». Les entreprises commerciales n'ont cessé de grandir jusqu'à coloniser tout l'espace et épuiser les ressources: "les pertes causées par les bulles financières lorsqu'elles éclatent dépassent désormais en taille la capacité d'absorption des Etats", ainsi que l'a montré l'ampleur inédite de la crise des subprimes de 2008. Livrant une vision quelque peu fossilisée de l'ESS, P. Jorion estime que cette forme d'économie, après avoir connu son apogée entre 1820 et 1850, a échoué à régler les questions de l'intérêt sur l'emprunt, de l'héritage et de la propriété. Il est urgent que l'ESS s'empare de ces sujets, car, "s'il s'agissait en 1848 d'un choix de société, ily va aujourd'hui de la survie de l'espèce».

Selon les économistes Philippe-Noël Giraud (Mines-Paris et Paris-Dauphine) et Florence Jany-Catrice (Clerse-CNRS), les indicateurs établis dans le contexte de la reconstruction de l'après-guerre continuent à imposer la dictature de la moyenne aux politiques publiques. Ils sont pourtant inefficients à mesurer les inégalités et le capital humain, de même que, comme l'a souligné Philippe Askenazy (CNRS, Cepremap), les référentiels classiques, donnés comme acquis et incontournables, ne sont en réalité que des principes idéologiques. Or ils génèrent des effets pervers, comme le retour de 
la rente accaparée par certains, l'impossibilité à penser des investissements de long terme et le fait que les marchés peuvent apporter des jugements sur les Etats, indépendamment de la volonté démocratique. Ricardo Petrella (université de Louvain, IERPE, Bruxelles) a fait un réquisitoire de l'actuelle financiarisation spéculative de la vie qui hypothèque l'avenir des jeunes générations ("le vol du futur»), ainsi que des politiques de privatisation qui ont attaqué les fondements des biens communs, incubateurs du "vivre ensemble». Or, non seulement ces derniers sont essentiels (l'eau notamment), mais ils sont aussi non substituables, universels et non territorialisables. Leur caractère public est incontestable, selon R. Petrella, qui se démarque ainsi quelque peu des thèses d'E. Ostrom. Dénonçant la volonté de rentabiliser les ressources, qu'il s'agisse de ressources naturelles ou " humaines", il a porté un regard critique sur un certain conformisme de l'ESS qui a amené mutuelles et coopératives, ces trente dernières années, à adopter le vocabulaire de l'entreprise illustrée par l'usage à tout va du terme "gouvernance».

\section{Les nouvelles voies possibles de l'ESS}

Face au constat partagé et désespérant d'une situation d'urgence planétaire, on attendait des participants qu'ils se livrent à une analyse prospective et proposent des solutions de sortie: comment "créer de nouvelles politiques vers une mondialisation de l'ESS».

Rosabeth Moss Kanter (Harvard Business School) s'est défendu d'entrée de chercher à renouveler la doctrine, préférant exposer avec enthousiasme un catalogue des pratiques vertueuses des fondations créées par les «entrepreneurs avec une conscience sociale », dont Bill Gates est évidemment le parangon. Cet entrepreneuriat social (social empowerment), qui est selon elle plus innovateur que les coopératives (conservatrices à cause de leur sociétariat passif), serait la nouvelle frontière... Ce dont l'auditoire n'a pas semblé convaincu. Paul Seabright (Toulouse School Economics) considère au contraire que l'ESS possède cette force spécifique de responsabiliser tous les acteurs, mais encore faudrait-il que les structures mises en place donnent aux gens l'envie de la découverte et de l'aventure. Gaël Giraud (CNRS) a affirmé que l'ESS a un rôle à jouer dans la mise en œuvre de la transition énergétique, comme la rénovation thermique des bâtiments ou l'éco-mobilité, deux chantiers majeurs non exposés à la concurrence internationale du fait qu'ils ne sont pas rentables à court terme et que les emplois ne sont pas délocalisables.

Rappelant la formule "Le lien doit précéder le bien " de Mauss, Jean-Louis Laville (Cnam) a esquissé trois scenarios d'évolution de l'ESS. Les deux premiers ne sont guère enthousiasmants: isomorphisme institutionnel (les entreprises de l'ESS devenant des prestataires de services publics) ou simple rôle d'amortisseur du capitalisme (sans proposition alternative). Le troisième, celui d'économie plurielle, est en voie d'expérimentation en Amérique latine. Il se traduit par un rapprochement de l'ESS avec de nouvelles formes d'économie territorialisée.

Cette ESS polymorphe, génératrice de nouvelles politiques publiques en Amérique latine, était justement au cœur du propos de Ruth Muñoz, chercheure à l'université nationale General-Sarmiento de Buenos Aires. Elle se caractérise par une grande diversité de pratiques, qu'il s'agisse des systèmes de troc (trueques) et des entreprises récupérées nées de la crise en Argentine au début des années 2000 ou de monnaies sociales au Brésil, où un secrétariat de l'ES a été créé en 2003 au ministère du Travail et de l'Emploi. L'organisation éco-communautaire appuyant les associations de petits producteurs est propre à la situation bolivienne depuis l'élection d'Evo Moralès, président indigène. L'évolution la plus aboutie est certainement celle 
de l'Equateur, avec la constitution de 2006 qui a fixé comme objectif le bien-être pour tous, en comptant pour y parvenir sur une économie plurielle où le secteur solidaire et populaire aurait toute sa place.

A l'issue de ces présentations, l'Amérique latine semble bien être actuellement le continent le plus en pointe pour le renouvellement de l'ESS. Cependant, celle-ci n'en reste pas moins vulnérable face aux aléas des changements politiques, comme le montre au Brésil l'abandon des budgets participatifs dans les municipalités perdues par le Parti des travailleurs.

Le ministre del'ESS, Benoît Hamon, a conclu, comme il se doit, cette journée en appelant à "déconstruire le catéchisme économique", à "vaincre les dogmes qui ont contribué à mener l'économie mondiale à sa perte». Il a rappelé la faculté de résilience à la crise qui caractérise les entreprises de l'ESS et a souligné l'importance de définir une stratégie de développement pour l'ESS, ce qui devrait se concrétiser en juin prochain par le vote d'une loi-cadre. "Replacer le citoyen au cœur des processus de décision économique » est une question de volonté politique, a-t-il affirmé. On espère que sa voix sera entendue au sein du gouvernement auquel il appartient.

PATRICIA TOUCAS-TruYEN

\section{L'ESS, un modèle économique à part entière pour les collectivités}

Le 5 mars, Marie-Noëlle Lienemann, au titre du groupe d'études sénatorial chargé de l'ESS et présidé par Marc Daunis, accueillait au Sénat les différents représentants d'associations d'élus de collectivités locales pour signer avec Benoît Hamon une déclaration commune, «L'ESS, un modèle économique à part entière, apportant des réponses aux besoins de nos territoires ", dont nous reproduisons ici de larges extraits.

"En cette période de crises financière, économique, sociale et écologique, en cette heure où il devient urgent de répondre à l'aspiration démocratique exprimée par nos concitoyens, l'économie sociale et solidaire démontre qu'il est possible d'entreprendre autrement, de produire et de consommer autrement, en respectant le salarié, le consommateur, le citoyen.

L'ESS représente plus de 2,3 millions d'emplois en France, et avec eux, davantage de cohésion sociale, d'innovations et de mieux-être au cœur des territoires. Elle est un modèle économique à part entière, apportant des réponses aux besoins de nos territoires. Elle ouvre de nouveaux espaces démocratiques, stimulant une éducation populaire et la formation des citoyens. [...] Nous saluons la loi en préparation sur l'économie sociale et solidaire qui doit permettre d'affirmer l'importance d'un développement socio-économique basé sur la coopération entre acteurs et sur l'ancrage territorial, plutôt que sur la mise en concurrence et la compétitivité [et] de mettre en évidence les valeurs de l'ESS, sa capacité à répondre collectivement à des besoins communs et sa dimension d'innovation sociale. L'économie sociale et solidaire n'est pas une démarche philanthropique ou le supplément d'âme des politiques publiques, elle doit avoir une place stratégique au cour de celles-ci et irriguer la plupart des politiques publiques. Elle réinterroge la création de richesse et notre manière de la comptabiliser et favorise une démocratie participative active. Les collectivités locales, acteurs majeurs du développement des territoires urbains et ruraux, organisatrices du dialogue de proximité avec la société civile, ont, aux côtés de l'Etat, un rôle fondamental afin de favoriser le développement de l'ESS dans les territoires. C'est la raison pour laquelle nous avons acté le principe d'une Rencontre annuelle des collectivités locales autour de l'ESS et nous nous engageons à poursuivre notre collaboration autour de cette question. Nous appelons l'Etat et ses représentants à: - affirmer la place de l'économie sociale et solidaire au sein de l'économie, à développer 
de façon interministérielle une ambitieuse politique publique d'économie sociale et solidaire, en collaboration avec les collectivités locales au travers par exemple de conventions pluriannuelles Etat-collectivités; - reconnaître la contribution des acteurs de l'ESS à l'intérêt général, à accompagner les acteurs territoriaux dans la mise en place des SIEG et des SSIG et à faire évoluer le Code des marchés publics, afin de mieux prendre en compte les acteurs de l'ESS, dans la suite des travaux actuellement menés à Bruxelles surcesujet;

- inscrire la promotion et le développement de l'ESS dans l'ensemble des schémas directeurs des politiques publiques [...];

- veiller à ce que la dimension ESS soit prise en compte dans tous les projets de loi relatifs au développement économique, à la démocratie locale, à la formation professionnelle, à l'éducation et à la recherche.

Christiane Bouchart, présidente du RTES; Marie-Guite Dufay, présidente de la commission ESS de l'Association des régions de France; Claudy Lebreton, président de l'Assemblée des départements de France; Jacques Pélissard, président de l'Association des maires de France; Lö̈c Cauret, vice-président de l'Assemblée des communautés de France, déléguéau développement économique et à l'emploi; Adeline Hazan, secrétaire générale de l'Association des maires des grandes villes de France. »

\section{Au Portugal, consensus politique autour de l'adoption de la loi-cadre}

L'économie sociale (ES) a acquis au Portugal sa première reconnaissance constitutionnelle au lendemain de la révolution des Eillets ${ }^{(3)}$. Cet outillage juridique initial a été ensuite consolidé par le Code coopératif et la loi sur les bénéfices fiscaux de 1980,

(3) Leite Joao Salazar, «L'économie sociale au Portugal », Recma, $\mathrm{n}^{\circ}$ 320, avril 2011, www.recma.org/node/1288. puis le décret sur les coopératives d'intérêt public (dites régies coopératives). L’adoption par l'Assemblée de la république à l'unanimité, le 15 mars, de la loi-cadre de l'économie sociale (LCES) ${ }^{(4)}$ constitue un nouveau tremplin pour l'essor de ces organisations dans ce pays cruellement touché par la crise.

Pour la Cases ${ }^{(5)}$, «il s'agit d'un signe donné à toute la communauté: il est possible en démocratie, sur la base du débat et du dialogue, d'établir des consensusfavorables au développement du pays ». De fait, l'une des particularités des législations portugaises relatives à l'économie coopérative et sociale est que, depuis la Constitution de la République et le Code de 1980, leur adoption a toujours fait consensus, transcendant les clivages politiques et idéologiques. La récente loi-cadre ne fait pas exception: les négociations ont été longues, mais tout s'est passé finalement comme si l'utilité de l'ES faisait l'unanimité, alors que le contexte politique portugais est actuellement très tendu. La droite au pouvoir a accepté des propositions d'amendement émanant des communistes, tandis que la gauche acceptait les amendements proposés par les partis du centre et de droite.

\section{Rapprocher les familles}

Le Portugal devient ainsi le second pays européen, après l'Espagne en mars $2011^{(6)}$, à adopter une loi-cadre pour l'ESS. Le texte adopté est finalement assez proche de la loi espagnole. Sa mise en application sous l'égide du Conseil national de l'économie sociale (Cnes) implique préalablement

(4) Consultable sur www.recma.org/node/3318.

(5) La Cooperativa António Sérgio para a Economia Social est une coopérative d'intérêt public qui a hérité des compétences de l'ancien institut public Inscoop. Elle a pour vocation de faire dialoguer les organisations de l'économie sociale et de leur faciliter l'accès à des activités de formation, au microcrédit, aux financements, etc. (6) Rafael Chaves, « La loi espagnole d'économie sociale: évaluation du point de vue de la politique publique ", Recma, n³21, juillet 2011, www.recma.org/node/1405. 
une révision législative des sous-secteurs qui devrait aboutir, au terme d'un processus d'harmonisation de six mois, à des solutions juridiques propices à l'épanouissement des trois familles de l'économie sociale. Il s'agit surtout de favoriser un rapprochement des trois branches - mutuelles, associations et coopératives - qui, comme en France, peinent souvent à travailler en synergie. Ce nouveau cadre juridique offre l'opportunité à tous les acteurs de l'ES de mutualiser leurs compétences pour entreprendre des réalisations conjointes, surtout au niveau local, en matière de lutte contre la pauvreté, d'insertion et d'innovations sociales. Il est prévu que les entreprises d'économie sociale siègent au Conseil économique et social avec les confédérations patronales et syndicales, et il serait souhaitable qu'elles puissent, à plus long terme, être représentées par une confédération nationale unique, du type de la Cepes espagnole.

\section{Un modèle à suivre en Europe}

Avec cette loi-cadre, adoptée dans le sillage de l'Espagne, le Portugal entend montrer la voie aux autres pays de l'Union européenne, qui en dotant l'ESS d'une législation spécifique et adaptée à ses besoins propres pourraient mettre un terme à sa dilution dans le secteur commercial lucratif au niveau de l'UE. Les partenaires européens ont ainsi été conviés à discuter de l'avenir du secteur dans le cadre d'une conférence internationale, qui aura lieu à Cascais fin juin 2013. En clair, la globalisation - fût-elle limitée à l'espace européen - ne peut valoir que pour les logiques capitalistes. Les entreprises de l'économie sociale, à visée humaniste et non lucrative, doivent être des acteurs de plein droit dans le dialogue civil et social, national et communautaire. Aux Etats membres de faire cet effort législatif national qui finira - espérons-le - par inverser l'ordre des priorités à Bruxelles.

\section{Une future loi-cadre pour l'économie sociale au Québec}

Le Québec compte environ 7000 entreprises d'économie sociale qui emploient 125000 personnes pour huit millions d'habitants. Celles-ci génèrent un bénéfice collectif de plus de 17 milliards de dollars, ce qui représente environ $8 \%$ du PIB de la province. Si l'économie sociale remonte à plus de cent cinquante ans ans dans la Belle Province, sa reconnaissance publique ne date que de la fin du $\mathrm{xx}^{\mathrm{e}}$ siècle. L'actuel examen d'une loi-cadre y représente, comme en France, un événement politique majeur.

\section{L’ESS au Québec: un secteur ancien mais mal identifié}

Au Québec, comme en Europe, l'économie sociale s'est déclinée sous les formes coopérative, mutualiste et associative. Ces différentes composantes fonctionnant dans leur cadre règlementaire respectif, il a fallu attendre le sommet socioéconomique de Montréal en $1996^{(7)}$ pour qu'émerge sur la scène publique le concept d'économie sociale. Le projet de loi-cadre sur l'économie sociale déposé à l'Assemblée nationale le 19 mars par le ministre des Affaires municipales, Sylvain Gaudreault, devrait contribuer à donner à ce secteur, aussi cloisonné qu'il peut l'être en France, une cohérence et une identité fondées sur des valeurs communes, tout en constituant un enjeu primordial pour la reconnaissance institutionnelle de l'importance de cette forme d'économie, au même titre que les économies publique et marchande.

\section{Une reconnaissance inédite}

Le projet de loi se fixe pour objectif de promouvoir l'économie sociale comme levier de développement socioéconomique, de soutenir le développement de l'économie

(7) Poirier Y., "Syndicalisme et ESS : l'expérience québécoise », Recma, n³22, octobre 2011. 
sociale par l'élaboration ou l'adaptation d'outils d'intervention, dans une perspective de cohérence gouvernementale et de transparence, et de favoriser l'accès aux mesures et aux programmes de l'administration pour les entreprises du secteur.

La mise en œuvre en sera confiée au Chantier de l'économie sociale et au Conseil québécois des coopératives et des mutuelles (CQCM) ${ }^{(8)}$, désignés comme les interlocuteurs privilégiés du gouvernement en matière d'économie sociale. Pour Louis Favreau, professeur à l'Uqam, cette reconnaissance du CQCM au même titre que celle du Chantier est positive, dans la mesure où elle tempère l'hégémonie de ce dernier souvent considéré, à tort, comme représentant l'ensemble du monde de l'économie sociale, alors qu'il représente davantage le monde associatif (qui se revendique de l'économie sociale) que coopératif et mutualiste (traditionnellement appelé économie solidaire au Québec).

\section{Une définition inclusive}

D'un point de vue pratique, le nouveau cadre juridique précisera les conditions fiscales d'attribution des subventions et des marchés publics pour les contrats passés entre l'Etat et les entreprises d'économie sociale, qui ne semblent pas avoir jusqu'alors bénéficié d'une fiscalité avantageuse, comme c'est le cas en France. De même, les entreprises relevant de l'économie sociale auraient accès à la future Banque de développement économique, au même titre que les entreprises capitalistes. En outre, le projet de loi accorde une place importante aux acteurs nationaux, régionaux et sectoriels, en prévoyant la création d'une table des partenaires en économie sociale. Deux spécialistes de l'ESS, Benoît Lévesque, professeur émérite à l'Uquam, et Claude Béland, président du mouvement «Démocratie et citoyenneté du Québec » et ancien

(8) Le Conseil québécois de la coopération et de la mutualité est l'organisateur de la conférence internationale organisée à Lévis en 2010 (lire " Le souffle coopératif québécois », «Actualité », Recma, nº 318, 2010). dirigeant du Mouvement Desjardins, expriment le souhait que cette loi retienne une définition inclusive de l'économie sociale: "Aucune coopérative, aucune mutuelle et aucune association ayant des activités économiques ne devraient être exclues si elles répondent aux caractéristiques retenues. On devrait également considérer la possibilité d'y inclure les entreprises ayant des statuts juridiques assimilables, en précisant les modalités de leur insertion. Cette ouverture devrait cependant s'accompagner de la possibilité pour certaines de s'exclure, même si elles en partagent toutes les caractéristiques communes. ${ }^{(9)}$ » Toutes ressemblances avec les orientations discutées de ce côté-ci de l'Atlantique (voir «En bref » de ce numéro) ne sont pas fortuites.

\section{L'ESS transatlantique}

Benoît Hamon, ministre délégué chargé de l'ESS, et Sylvain Gaudreault, ministre des Affaires municipales du Québec, ont signéle 15 mars 2013 une entente franco-québécoise en matière d'ESS, faisant suite à une déclaration d'intention adoptée en octobre 2012, à l'occasion du Sommet international des coopératives à Québec. Planchant simultanément sur la consolidation juridique du secteur de l'ESS, la France et la province canadienne cherchent à développer les échanges d'expériences, d'expertises et de bonnes pratiques et à promouvoir l'ESS dans le monde de la francophonie.

Plus concrètement, la coopération doit porter sur les outils de financement et de promotion de l'ESS, ainsi que sur l'enseignement et la formation. La diffusion sur le Web de la conférence organisée par le Chantier de l'économie sociale le 30 janvier dernier (disponible sur www.webtv.coop) rend compte des similitudes et des différences de l'ESS de part et d'autre de l'Atlantique et des apports réciproques qui pourront être tirés de cette entente au sommet.

PATRICIA TOUCAS-TRUYEN 


\section{Quelle économie sociale en Europe?}

Espagne, Portugal, France bientôt: l'économie sociale (et solidaire) progresse sur le plan législatif national; au Parlement européen également, où il est de nouveau question d'un statut de mutualité européenne et où les députés viennent d'adopter le premier texte en matière de finance solidaire et d'entrepreneuriat social. Deux textes importants et deux conceptions sensiblement différentes de l'économie sociale.

\section{Le retour du statut}

\section{de mutuelle européenne}

Le Parlement européen a adopté le 14 mars, en séance plénière, le rapport d'initiative législatif de l'italien Luigi Berlinguer sur le statut de mutuelle européenne. Il s'agit de demander à la Commission européenne (qui avait enterré le sujet en septembre 2005) de faire une proposition législative en vue de créer ce statut, véritable serpent de mer depuis des années. "Nous avons besoin de ce statut. La commission doit agir, il faut mettre un terme à toute tergiversation ", a défendu Luigi Berlinguer avant le vote. L'intérêt de ce texte (consultable sur www. recma.org/node/3320) réside notamment dans la réapparition de la locution, pourtant très peu européenne, d'économie sociale (considérant F), définie comme des " groupes volontaires de personnes physiques ou morales dont l'objectifest de répondre aux besoins de leurs membres plutôt que d'obtenir un retour sur investissement ». Le même considérant $(\mathrm{H})$ poursuit en affirmant que ces organisations "fonctionnent conformément aux principes de l'affiliation ouverte et volontaire et de la solidaritéentre membres et sont gérées selon des principes démocratiques (tels que le principe "Un membre, une voix”) » et contribuent "à une gestion responsable et durable ». Les parlementaires défendent par ailleurs une conception très large de la mutualité, qui peut "couvrir tous types de risques patrimoniaux et de risques de vie», ainsi que "fournir des services dans d'autres domaines, tels que le logement ou le crédit ». Après avoir rappelé le poids de la mutualité en Europe (25\% du marché de l'assurance et $70 \%$ du nombre total d'entreprises du secteur), toujours ignorée du marché unique, les parlementaires européens demandent donc que ces entreprises bénéficient "d'un statut européen qui les mette sur un pied d'égalité avec les autres formes d'entreprises dans l'Union ». D'autant plus que le secteur privé est appelé à contribuer à seconder plus massivement encore des systèmes de protection sociale exsangues. Or, les mutuelles, qui «n'ont pas d'actions » et dont "les bénéfices sont plutôt réinvestis que distribués aux membres", doivent être soutenues pour défendre le modèle social européen. Car il y a lieu "d'empêcher les mutualités de prendre, en vue de rester compétitives, des mesures qui les rendraient semblables à leurs homologues commerciales, par exemple en introduisant la sélection des risques ou des critères plus stricts d'adhésion, voire en émettant des actions afin d'accroître leurs marges de solvabilité ». La Commission européenne n'a pas donné son avis, mais elle avait lancé quelques jours avant le vote du Parlement une consultation publique sur ce projet de statut. Les réponses au questionnaire devront parvenir avant le 14 juin 2013.

\section{Des fonds d'entrepreneuriat social européens}

Deux jours plus tôt, le 12 mars, le même Parlement européen adoptait le premier texte législatif en matière de finance solidaire et d'entrepreneuriat social (www. recma.org/node/3334). Ce projet de règlement, voté à une très large majorité, créé un nouvel outil de financement pour les entreprises sociales. Le règlement autorise les banques et les assurances à créer des fonds d'investissement et à les appeler "fonds d'entrepreneuriat social européens » (Fese ou Eusef en anglais). Devront être investis dans des entreprises sociales $70 \% \mathrm{du}$ 
capital de ces fonds (en France, les fonds solidaires doivent y consacrer $90 \%$ ), et la somme investie doit être au minimum de 100000 euros. Ce projet doit encore être validé par le Conseil européen. La signature officielle est prévue en avril.

La définition des entreprises sociales retenue par les députés européens précise qu'il s'agit de structures ayant " pour objectif principal [...] de produire des effets sociaux positifs et mesurables ». Il s'agit donc de fournir "des biens ou des services à des personnes vulnérables, marginalisées, défavorisées ou exclues ». La définition porte ainsi davantage sur le bénéficiaire final (personnes vulnérables, marginalisées, défavorisées ou exclues) que sur les règles régissant ces structures.

Les parlementaires européens ont pris soin d'inclure les financeurs solidaires, ce qui permettra à des structures comme France active de bénéficier des fonds, sans exclure les organismes financiers les moins « d'économie sociale" au sens débattu par ces mêmes eurodéputés deux jours plus tard... 